\title{
Startup process in the Richtmyer-Meshkov instability
}

\author{
M. Lombardinia) and D. I. Pullin \\ Graduate Aeronautical Laboratories, California Institute of Technology, Pasadena, California 91125, USA
}

(Received 2 October 2008; accepted 4 November 2008; published online 14 April 2009)

\begin{abstract}
An analytical model for the initial growth period of the planar Richtmyer-Meshkov instability is presented for the case of a reflected shock, which corresponds in general to light-to-heavy interactions. The model captures the main features of the interfacial perturbation growth before the regime with linear growth in time is attained. The analysis provides a characteristic time scale $\tau$ for the startup phase of the instability, expressed explicitly as a function of the perturbation wavenumber $k$, the algebraic transmitted and reflected shock speeds $U_{S_{1}}<0$ and $U_{S_{2}}>0$ (defined in the frame of the accelerated interface), and the postshock Atwood number $A^{+}$: $\tau=\left[\left(1-A^{+}\right) / U_{S_{2}}+\left(1+A^{+}\right) /\left(-U_{S_{1}}\right)\right] /(2 k)$. Results are compared with computations obtained from two-dimensional highly resolved numerical simulations over a wide range of incident shock strengths $S$ and preshock Atwood ratios $A$. An interesting observation shows that, within this model, the amplitude of small perturbations across a light-to-heavy interface evolves quadratically in time (and not linearly) in the limit $A \rightarrow 1^{-}$. (C) 2009 American Institute of Physics.
\end{abstract}

[DOI: 10.1063/1.3091943]

\section{INTRODUCTION}

The Richtmyer-Meshkov instability (RMI) arises when a shock wave interacts with a perturbed interface separating two fluids of different densities. It combines different phenomena such as, but not limited to, shock refraction, hydrodynamic stability, and both linear and nonlinear growth periods. Such instability occurs in a wide variety of applications ranging from astrophysics to inertial confinement fusion, including multiphase and reacting flows. Here and hereinafter, we consider only the case of a reflected shock corresponding in general to a "light $\rightarrow$ heavy" shockcontact refraction.

There are two important contributions to the early time, or small-amplitude linear growth of the instability, before nonlinear development of the perturbation appears. First, the baroclinic deposition of vorticity due to the direct interaction of the incident shock with the interface, when the pressure gradient at the shock is misaligned with the local density gradient at the interface. If the initial interface is sharp, it can therefore be viewed as a vortex sheet that leads to its own self-induced distortion. The second contribution concerns the influence of the transmitted and reflected shocks as they leave density and vorticity perturbations behind them. Relaxation of these shock fronts both deposits bulk vorticity and emits acoustic waves that, by reverberation, modifies the vorticity distribution on the interface. In the weak shock limit, the linear growth reduces essentially to the first contribution, while for strong incident shocks, the produced transmitted shock takes longer time to separate from the interface.

Richtmyer first derived the compressible perturbed equations and obtained a simple analytical expression for the asymptotic linear growth rate, ${ }^{1}$ assuming that transmitted and reflected shocks have traveled sufficiently far, compared to the wavelength of the perturbation, that the second contribu-

a)Electronic mail: manuel@caltech.edu. tion is subdominant. Other methods concentrating also on the first contribution have attempted to correct the impulsive growth rate to better model the behavior for strong incident shocks or high Atwood ratio without loss of simplicity. ${ }^{2} \mathrm{Be}-$ sides, numericists and experimentalists addressed the effect of shock proximity by using empirical corrections to the impulsive growth rate. ${ }^{3-5}$ More complex, semianalytical studies have taken into account all relevant phenomena, ${ }^{6,7}$ and showed good agreement with numerical results obtained by linearizing the Euler equations between the perturbed interface and transmitted/reflected waves, ${ }^{8}$ and with the linear interaction analysis at low Atwood numbers of Griffond. ${ }^{9}$

In what follows, by modeling the proximity of the receding transmitted and reflected shocks, the analysis in Sec. II establishes a simple analytical expression for the growth rate that captures some of the early features of the perturbation evolution before it has reached the asymptotic growth linear in time. As analyzed in Sec. III, the solution addresses the early-time physics of the linear growth, with a characteristic time $\tau$, while allowing for the determination of the asymptotic, or later-time, growth rate by additional physics. Section IV compares results to computations obtained from two-dimensional numerical simulations of the RMI under various initial conditions. Different realistic combinations of Atwood ratio and specific heat ratios are tested, as well as incident shock strength, initial perturbation amplitude, and wavenumber. A more thorough parametric study of the characteristic time $\tau$ is presented in Sec. V.

\section{ANALYTICAL MODEL}

\section{A. General formulation}

At $t=0$, in Cartesian axes $(x-z)$, a plane shock traveling to the left (negative $z$-direction) impacts a plane unperturbed density interface, $z=0$, separating two fluids of different density, producing a transmitted shock, and a reflected shock. 


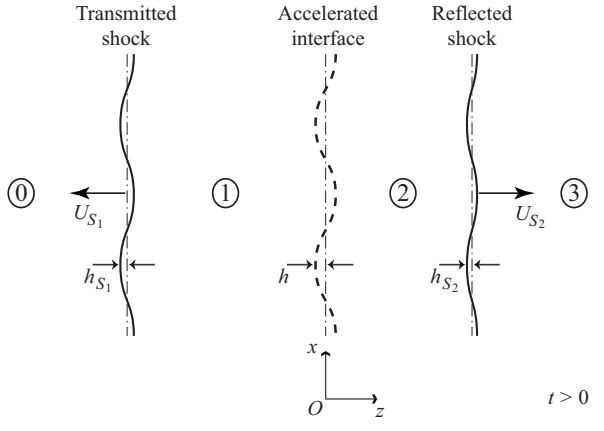

FIG. 1. Perturbed system.

We work in a frame of reference in which the undisturbed interface is always at $z=0$, and in which the transmitted shock velocity is $U_{S_{1}}<0$ and the reflected shock velocity is $U_{S_{2}}>0$. We define more generally $S_{j}(t), j=1,2$, as the average position of these shocks at time $t$. In the laboratory reference frame, the interface is impulsively accelerated to velocity $-\Delta W \mathbf{e}_{z}, \Delta W>0$, at $t=0$. For given fluids and given incident shock strength, $U_{S_{1}}, U_{S_{2}}, \Delta W$, and the postshock densities and Atwood number $A^{+}$can be determined by solution of a one-dimensional Riemann problem. We will focus on regions directly in contact with the interface and denote these by the subscript $j$, where $j=1$ for the region to the left, $z<0$, of the interface and $j=2$ for the region to the right, $z>0$, of the interface. The interface is slightly perturbed (Fig. 1), with a perturbation amplitude small compared to its fundamental wavelength. Its position is

$$
z=\zeta(x, t)=h(t) e^{\imath k x} .
$$

The purpose of this analytical work is to determine a simple approximation for the growth $h(t)$ for small $t$ within the linear approximation.

The fundamental length scale in this problem is the wavelength of the perturbation $2 \pi / k$. We choose $1 / k$ as our characteristic length scale. The characteristic velocity scale is chosen to be $k h(0) /(2 \pi) A^{+} \Delta W$ since Richtmyer's impulsive model of the asymptotic growth rate for the linear RMI (Ref. $1)$ is $k h(0) /(2 \pi) A^{+} \Delta W$, with $A^{+}=\left(\rho_{1}-\rho_{2}\right) /\left(\rho_{1}+\rho_{2}\right)$ the postshock Atwood ratio based on postshock densities and $h(0)$ amplitude of the perturbation at $l=0$. An appropriate time scale characteristic of the linear growth of the instability is thus $2 \pi /\left[k^{2} h(0) A^{+} \Delta W\right]\left(>0\right.$ as long as $\left.A^{+}>0\right)$. The density scales like the constant postshock densities $\rho_{1}$ to the left of the interface, or $\rho_{2}$ to the right. Similarly, the pressure scales like $\rho_{j}\left[k h(0) /(2 \pi) A^{+} \Delta W\right]^{2}, j=(1,2)$. The impulsive acceleration $g=\Delta W \delta_{D}(t)$ of the interface, where $\delta_{D}$ is the Dirac delta function, is made dimensionless by the characteristic acceleration $k\left[k h(0) /(2 \pi) A^{+} \Delta W\right]^{2}$. Define $a_{j}$ as the postshock speed of sound to the left or right of the interface. To summarize, for each region $j=(1,2)$, original quantities are related to dimensionless quantities, denoted with a bar, as follows:

$$
\begin{aligned}
& \boldsymbol{x}=\frac{1}{k} \overline{\boldsymbol{x}}, \quad t=\frac{1}{k \varepsilon_{j} a_{j}} \bar{t}_{j}, \quad \varrho_{j}=\rho_{j} \varrho_{j}, \quad \boldsymbol{u}_{j}=\varepsilon_{j} a_{j} \overline{\boldsymbol{u}}_{j}, \\
& p_{j}=\rho_{j}\left(\varepsilon_{j} a_{j}\right)^{2} \bar{p}_{j}, \quad g=k\left(\varepsilon_{j} a_{j}\right)^{2} \bar{g}_{j},
\end{aligned}
$$

where the following dimensionless parameter is defined as

$$
\varepsilon_{j}=\frac{k h(0)}{2 \pi} A^{+} \frac{\Delta W}{a_{j}} .
$$

The parameter $\varepsilon_{j}$ defined in Eq. (3) is in general small since $k h(0) /(2 \pi) \ll 1, A^{+} \leqslant 1$, and $\Delta W / a_{j}$ is of the order of unity. This latter ratio can be greater than unity in the heavy region 1 when the incident shock Mach number $M_{I}$ is very high but remains less than 10 as long as $A^{+}$is not too close to unity and the incident shock is not too strong. For example, in the case of a "light air $\rightarrow$ heavy $\mathrm{SF}_{6}$ " shock interaction at $M_{I}=8.0$, whose Atwood number $A^{+} \simeq 0.7$ is quite high ( $1: 5$ density ratio) in the heavy region $\Delta W / a_{1} \simeq 4.2$.

The Euler equations in the frame of the accelerated interface are now written in terms of dimensionless quantities on each side of the interface,

$$
\begin{aligned}
& \frac{\partial \bar{\varrho}_{j}}{\partial \bar{t}_{j}}+\frac{\partial}{\partial \bar{x}}\left(\bar{\varrho}_{j} \bar{u}_{j}\right)+\frac{\partial}{\partial \bar{z}}\left(\bar{\varrho}_{j} \bar{w}_{j}\right)=0, \\
& \bar{\varrho}_{j}\left(\frac{\partial \bar{u}_{j}}{\partial \bar{t}_{j}}+\bar{u}_{j} \frac{\partial \bar{u}_{j}}{\partial \bar{x}}+\bar{w}_{j} \frac{\partial \bar{u}_{j}}{\partial \bar{z}}\right)=-\frac{\partial \bar{p}_{j}}{\partial \bar{x}}, \\
& \bar{\varrho}_{j}\left(\frac{\partial \bar{w}_{j}}{\partial \bar{t}_{j}}+\bar{u}_{j} \frac{\partial \bar{w}_{j}}{\partial \bar{x}}+\bar{w}_{j} \frac{\partial \bar{w}_{j}}{\partial \bar{z}}\right)=-\frac{\partial \bar{p}_{j}}{\partial \bar{z}}+\bar{\varrho}_{j} \bar{g}_{j}\left(\bar{t}_{j}\right), \\
& \left(\frac{\partial \bar{p}_{j}}{\partial \bar{t}_{j}}+\bar{u}_{j} \frac{\partial \bar{p}_{j}}{\partial \bar{x}}+\bar{w}_{j} \frac{\partial \bar{p}_{j}}{\partial \bar{z}}\right)=-\frac{1}{\varepsilon_{j}^{2}} \bar{\varrho}_{j}\left(\frac{\partial \bar{u}_{j}}{\partial \bar{x}}+\frac{\partial \bar{w}_{j}}{\partial \bar{z}}\right),
\end{aligned}
$$

where it has been assumed that the sound speed is uniform and constant equal to $a_{j}$. The acceleration of the frame in which the Euler equations are written is contained in the last term of Eq. (4c).

\section{B. Base flow and perturbations}

The flow is decomposed as a base part uniform in the transverse direction $x$ and a small perturbation sinusoidal in $x$. For any dimensionless flow quantity $\bar{q}_{j}$ in each region,

$$
\bar{q}_{j}\left(\bar{x}, \bar{z}, \bar{t}_{j}\right)=\bar{q}_{j}^{(0)}\left(\bar{z}, \bar{t}_{j}\right)+\varepsilon_{j} \bar{q}_{j}^{(1)}\left(\bar{z}, \bar{t}_{j}\right) e^{\imath \bar{x}}+\cdots .
$$

An admissible base flow in regions 1 and 2 is expressed as

$$
\bar{\varrho}_{j}^{(0)}=1, \quad \bar{u}_{j}^{(0)}=0, \quad \bar{w}_{j}^{(0)}=0, \quad \bar{p}_{j}^{(0)}=\bar{P}_{0}+\bar{g}_{j}\left(\bar{t}_{j}\right) \bar{z},
$$

where $\bar{P}_{0}$ is an arbitrary background pressure. The base flow is simply the incompressible response to an impulse that instantaneously accelerates the flow to the speed $\Delta W$ in the negative $z$-direction. Expressed in dimensional variables, the base quantities can be determined by solving the onedimensional Riemann problem of the shock interaction at $t=0$. Using Eqs. (5) and (6) in Eq. (4) for each region $j$, expanding all terms in $\varepsilon_{j}$ except the frame acceleration in Eq. 
(4c), and retaining quantities of the order of $\varepsilon_{j}$ then gives the $\mathcal{O}\left(\varepsilon_{j}\right)$ perturbation equations as

$\frac{\partial \bar{\varrho}_{j}^{(1)}}{\partial \bar{t}_{j}}+\iota \bar{u}_{j}^{(1)}+\frac{\partial \bar{w}_{j}^{(1)}}{\partial \bar{z}}=0$,

$\frac{\partial \bar{u}_{j}^{(1)}}{\partial \bar{t}_{j}}=-\iota \bar{p}_{j}^{(1)}$

$\rho_{j} \frac{\partial \bar{w}_{j}^{(1)}}{\partial \bar{t}_{j}} e^{l \bar{x}}=-\rho_{j} \frac{\partial \bar{p}_{j}^{(1)}}{\partial \bar{z}} e^{c \bar{x}}+\left(\rho_{2}-\rho_{1}\right) \bar{g}_{j}\left(\bar{t}_{j}\right)[H(\bar{z})-H(\bar{z}-\bar{\zeta})]$,

$\iota \bar{u}_{j}^{(1)}+\frac{\partial \bar{w}_{j}^{(1)}}{\partial \bar{z}}=0$,

where $H$ is the Heaviside function. Equation (7c) is obtained by subtracting Eq. (4c) from its equivalent equation for the base field, at every location $(x, z)$. Equation $(7 d)$ shows that the leading-order perturbed flow is incompressible and, from Eq. (7a), the perturbed density is independent of time. From Eqs. (7b) and (7c), the postshock perturbed flow is irrotational on each side of the interface except in a small window between $z=0$ and the interface $z=\zeta$. Therefore a perturbed potential $\bar{\phi}_{j}^{(1)}$ can be introduced such that $\overline{\boldsymbol{u}}_{j}^{(1)}=\overline{\nabla \phi_{j}^{(1)}}$. Outside of the forced region, the perturbed potential is a solution of the Laplace equation and can be written in terms of dimensional variables as

$$
\phi_{j}^{(1)}(z, t)=A_{j}(t) \sinh (k z)+B_{j}(t) \cosh (k z),
$$

where $A_{j}$ and $B_{j}$ are complex coefficients. The assumption of potential incompressible flow on each side of the interface was initially used by Layzer to describe the single-mode nonlinear growth. ${ }^{10}$ Most recently, based on Layzer's model at an infinite density ratio $(A=1)$ (see also Ref. 11), Srebro et al. found a general buoyancy-drag model at every $A$ describing the stages of the RMI and Rayleigh-Taylor instability. ${ }^{12}$ These models allows one to compute the bubble velocities, assuming that the flow is governed by the behavior near the bubble tips, supposed parabolic in shape.

\section{Boundary conditions at the shocks}

Boundary conditions at the transmitted and reflected shocks are now investigated. From the shock refraction process occurring when the incident shock impacts a perturbed interface with wavenumber $k$, the transmitted and reflected shock waves produced are similarly perturbed with the same wavenumber but are expected to evolve with a different growth than that of the interface perturbation. The initial shock perturbation $h_{S_{j}}\left(t=0^{+}\right)$is expected a priori of the same order of the amplitude of the interface perturbation $h\left(t=0^{+}\right)$. Our simulations (see Sec. IV) confirm the well-known results on planar shock stability: small perturbations at the shock front decay, while interface perturbations grow for $t>0$. Landau determined that the stability criterion for small disturbances traveling in the direction perpendicular to the shock was simply a consequence of the requirement of the second law of thermodynamics. ${ }^{13}$ Considering a corrugation in the transverse direction, Dýakov's criterion expresses that, in the case of a perfect gas, a discontinuous shock front is unconditionally stable. ${ }^{14}$ To complete the discussion, we note that Yang et al. found, in the case (not considered presently) of a reflected rarefaction wave, that the trailing edge of the fan is always unstable (Sec. III in Ref. 8).

Linearizing the Rankine-Hugoniot jump conditions with respect to small shock perturbation amplitude $h_{S_{j}}(t)$, i.e., corresponding to small interface perturbation $h(t)$, the axial velocity perturbation behind the shock can be related to $h_{S_{j}}(t)$ as

$$
w_{j}^{(1)}\left(S_{j}(t)+h_{S_{j}}(t) e^{\imath k x}, t\right) \simeq w_{j}^{(1)}\left(S_{j}(t), t\right) \simeq C_{j} \dot{h}_{S_{j}},
$$

where $C_{j}$ is a constant function of the unperturbed densities upstream and downstream of each shock. As the shock perturbation decays, the growth $\dot{h}_{S_{j}}$ tends to zero. We therefore assume the following approximate boundary condition: for $t>0$, the growth of the shock perturbation is zero at leading order in $\varepsilon_{j}$ and, therefore, the perturbed axial velocity must be zero at the shock. This assumption is consistent with incompressible, irrotational motion for the perturbed flow at leading order: the shocks then physically behave like moving plane boundaries along which there exists a uniform distribution of sources of just sufficient strength to produce the postshock incompressible flow. At higher order these "walls" essentially confine the reach of reverberating waves to the flow regions between the interface and the receding shocks. It is this effect that presently modifies Richtmyer's theory.

With this ansatz, Eq. (8) simplifies and we can express the perturbed velocity field in each region as

$$
\begin{aligned}
& u_{j}^{(1)}(z, t)=\iota k E_{j}(t) \cosh \left\{k\left[z-S_{j}(t)\right]\right\}, \\
& w_{j}^{(1)}(z, t)=k E_{j}(t) \sinh \left\{k\left[z-S_{j}(t)\right]\right\},
\end{aligned}
$$

where $E_{j}(t)$ are functions to be determined. This solution allows for shear at the "wall shocks" where the perturbed transverse velocity is nonzero. From the momentum equation in the $x$-direction, the pressure perturbation is

$$
p_{j}^{(1)}(z, t)=-\rho_{j} \frac{\partial}{\partial t}\left(E_{j}(t) \cosh \left\{k\left[z-S_{j}(t)\right]\right\}\right) .
$$

\section{Kinematic and dynamic conditions at the interface}

At the contact $z=\zeta(x, t)$, the perturbed $z$-velocity must be continuous, and, after linearization

$$
w_{1}^{(1)}(0, t) \simeq w_{2}^{(1)}(0, t) \simeq \dot{h} .
$$

Using the simplified form of $w_{j}^{(1)}(z, t)$ in regions 1 and 2 found in the previous paragraph, the linearized kinematic condition becomes

$$
-k E_{1}(t) \sinh \left[k S_{1}(t)\right]=-k E_{2}(t) \sinh \left[k S_{2}(t)\right]=\dot{h} .
$$

We observe that the coefficients $E_{j}$ are real. A dynamic boundary condition is obtained by integrating the perturbed 
momentum equation (7c) in $\bar{z}$ from $\bar{z}=-k h(t)$ to $k h(t)$. Observing that

$$
\int_{-k h}^{k h}[H(\bar{z})-H(\bar{z}-\bar{\zeta})] d \bar{z}=\bar{\zeta},
$$

the linearized dynamic condition to order $\varepsilon_{j}$ requires that at $z=h$ (in dimensional variables)

$$
p_{2}^{(1)}(-h, t)-p_{1}^{(1)}(h, t) \simeq\left(\rho_{2}-\rho_{1}\right) \Delta W \delta_{D}(t) h(t) .
$$

Using Eq. (11), the linearized dynamic condition for small perturbation amplitude $h$ becomes

$$
\begin{aligned}
-\rho_{2} & \frac{\partial}{\partial t}\left\{E_{2}(t) \cosh \left[k S_{2}(t)\right]\right\}+\rho_{1} \frac{\partial}{\partial t}\left\{E_{1}(t) \cosh \left[k S_{1}(t)\right]\right\} \\
& =\left(\rho_{2}-\rho_{1}\right) \Delta W \delta_{D}(t) h(t) .
\end{aligned}
$$

The functions $E_{j}(t)$ are fully determined, up to the initial condition $E_{j}(0)$, by integrating Eq. (16) from $t=0$ to $t$ and using the trivial condition $S_{j}(0)=0$. From Eq. (13), an expression for the functions $E_{j}$ and the growth rate $\dot{h}$ is found as

$$
\begin{aligned}
& E_{j}(t)=-\frac{2 \dot{h}_{\infty}}{k \sinh \left[k S_{j}(t)\right]\left(\left(1-A^{+}\right) \operatorname{coth}\left[k S_{2}(t)\right]+\left(1+A^{+}\right) \operatorname{coth}\left\{k\left[-S_{1}(t)\right]\right\}\right)}, \\
& \dot{h}(t)=\frac{2 \dot{h}_{\infty}}{\left(1-A^{+}\right) \operatorname{coth}\left[k S_{2}(t)\right]+\left(1+A^{+}\right) \operatorname{coth}\left\{k\left[-S_{1}(t)\right]\right\}}, \\
& \dot{h}_{\infty}=k h(0) A^{+} \Delta W H(t)+\frac{k}{2}\left[\left(1-A^{+}\right) E_{2}(0)-\left(1+A^{+}\right) E_{1}(0)\right] .
\end{aligned}
$$

The asymptotic growth rate predicted by Richtmyer is recovered in the first term of the right-hand side of Eq. (17c). The second term is a correction to Richtmyer's asymptotic growth rate depending on the coefficients $E_{j}(0)$ that still need to be modeled. This is discussed later in Sec. III C. The perturbation amplitude $h(0)$ at $t=0$ just as the shock passes the interface has not been clearly defined at this point; for example, $h(0)$ could be modeled as the arithmetic average of the preshock and postshock amplitudes $h\left(0^{-}\right) \equiv h_{0}$ and $h\left(0^{+}\right)$.

An alternative to the above analysis is to eliminate the functions $E_{j}$ from Eq. (16) using Eq. (13). This leads to the following second-order ordinary differential equation (ODE) in $h(t)$ :

$\ddot{h}=k F(t) \dot{h}$,

$F(t)$

$$
=\frac{\left(1-A^{\prime}\right) \dot{S}_{2}(t) \operatorname{csch}^{2}\left[k S_{2}(t)\right]-\left(1+A^{+}\right) \dot{S}_{1}(t) \operatorname{csch}^{2}\left[k S_{1}(t)\right]}{\left(1-A^{+}\right) \operatorname{coth}\left[k S_{2}(t)\right]-\left(1+A^{+}\right) \operatorname{coth}\left[k S_{1}(t)\right]} .
$$

Near $t=0$, assuming $S_{j}(t) \sim t$, Eq. (18b) shows that

$$
k F(t)=\frac{1}{t}+\mathcal{O}(t) .
$$

Therefore, the ODE (18a) has a regular singular point at $t$ $=0$. This admits a family of solutions, regular at $t=0$, with a single free parameter $\ddot{h}\left(0^{+}\right)$, which, from Eq. (17b), is directly related to the free asymptotic growth rate $\dot{h}_{\infty}$.

\section{STARTUP PERIOD FOR RMI}

\section{A. Startup time $\tau$}

First, we observe that, within the linearized approximation, the asymptotic growth rate $\dot{h}_{\infty}$, or equivalently, $\ddot{h}\left(0^{+}\right)$, is not determined presently. Once $\dot{h}_{\infty}$ is determined, the amplitude of the interface perturbation can be obtained by numerically integrating Eq. (17), given $h(0)$. Second, both terms in the denominator of Eq. (17c) are positive since $-1<A^{+}<1$, and $S_{1}(t)<0$ and $S_{2}(t)>0$. Third, the actual forms of $S_{1}(t)$ and $S_{2}(t)$ have not been used, and the instantaneous growth rate is a function only of the relative locations of the shocks and the interface. Since the leading order perturbed equations are incompressible, this suggests that our results are dependent on the equation of state of the fluids via the solution of the one-dimensional Riemann problem.

The shock locations can now be modeled with

$$
S_{j}(t)=U_{S_{j}} t H(t) .
$$

As $t \rightarrow 0^{+}$, the growth rate simplifies to

$$
\dot{h}=\dot{h}_{\infty} \frac{t}{\tau}+\mathcal{O}\left(t^{2}\right),
$$

where

$$
\tau=\frac{1}{2 k}\left(\frac{1-A^{+}}{U_{S_{2}}}+\frac{1+A^{+}}{\left(-U_{S_{1}}\right)}\right) .
$$

The equivalence $\ddot{h}\left(0^{+}\right)=\dot{h}_{\infty} / \tau$ is apparent from Eqs. (21) and (22). We notice that the model gives a nonzero positive ini- 
tial acceleration as $\tau>0$ and $\dot{h}_{\infty}>0$. The time $\tau$ obtained from our model represents the characteristic time during which the presence of the shocks influences the growth of the interface and is fully determined by solving the onedimensional Riemann problem described as the base flow. For $t \gg \tau$, the asymptotic growth $\dot{h}_{\infty}$ is recovered. Equation (21) shows that the growth rate immediately following the shock interaction, $\dot{h}\left(0^{+}\right)$, is zero. Numerical two-dimensional simulations shown later, as well as the linear compressible simulations of Yang et al. ${ }^{8}$ corroborate this observation. The kinematic condition (12) taken for $t \rightarrow 0^{+}$implies that $w_{j}^{(1)}$ $\left(z=0, t=0^{+}\right)$is zero, which is consistent with the boundary condition assuming zero axial velocity perturbation at the bifurcated shocks, the shocks being concentrated at $z=0$ as $t \rightarrow 0^{+}$.

\section{B. Asymptotic growth rate}

The growth rate, initially zero, increases to an asymptotic limit $\dot{h}_{\infty}$ as the shocks recede. Richtmyer ${ }^{1}$ initially proposed the impulsive model in an unbounded domain that gives the asymptotic growth as

$$
\dot{h}_{\text {Rich. }}=k h(0) A^{+} \Delta W .
$$

For any time, the transmitted and reflected waves are already at infinity. However, it has been shown by Yang et al. ${ }^{8}$ and confirmed by Wouchuk's semianalytical model ${ }^{7}$ that the impulsive model generally fails to represent the correct terminal linear growth rate. Solving the linearized Euler equations numerically, Yang et al. concluded that the linear theory and the impulsive model agree when the incident shock remains weak, but that large discrepancies appear for high incident shock strengths. Indeed, the simple observation that, for strong shocks, both shocked interface and transmitted shock remain close to each other for small $t$ is sufficient to contradict the assumption of shocks at infinity during the initial growth phase. Yang et al. also observed that the agreement with Richtmyer's model improves as the adiabatic exponents increase while remaining very close. Figure 16 in Ref. 8 (reflected shock case) shows that, for the air $\rightarrow \mathrm{SF}_{6}$ case, which we chose to study in detail in Sec. IV, the disagreement with Richtmyer's mode grows as the incident shock strength increases. A heuristic correction to the impulsive model has been proposed by Vandeboomgaerde $e t$ al. ${ }^{2}$ using averages of the pre- and postshock properties. However, as other impulsive formulations, the discrepancy with the exact solution can be very large as the incident shock becomes stronger. The true asymptotic growth rate will now be written as a correction to Richtmyer's asymptotic growth under the following form:

$$
\dot{h}_{\infty}=\mathfrak{F} \dot{h}_{\text {Rich. }} .
$$

In the limit of weak incident shock, $\mathfrak{F}$ is expected to tend to 1. Figure 16 in Ref. 8 represents the quantity $1 / \mathfrak{F}_{\text {Yang }}-1$ computed from the numerical computations of Yang et al.

\section{Initial tangential velocity at interface}

We briefly discuss a framework for modeling the terminal or long-time linear growth rate. From Eq. (17c), the terminal growth rate is determined up to the knowledge of the $E_{j}(0)$, in other words, the initial transverse velocities. According to Eq. (10a), in the case of zero initial transverse velocity on each side of the interface, Richtmyer's asymptotic solution is recovered. To improve the asymptotic model, a relationship is required between the asymptotic growth rate $\dot{h}_{\infty}$ and the initial jump in transverse velocity, or circulation distribution, across the interface. From Eq. (17b) substituted into Eq. (13) and then Eq. (10a), it can be shown that

$$
\begin{aligned}
& \dot{h}_{\infty}=-\imath \frac{\left(1-A^{+}\right) U_{S_{2}}-\left(1+A^{+}\right) U_{S_{1}}}{U_{S_{2}}-U_{S_{1}}} \Delta\left[u^{(1)}\right], \\
& \Delta\left[u^{(1)}\right] \equiv\left(u_{1}^{(1)}-u_{2}^{(1)}\right)_{t=0} .
\end{aligned}
$$

Here $\Delta\left[u^{(1)}\right]$ is the tangential velocity jump across the interface as $t \rightarrow 0^{+}$. In our notation it is purely imaginary, owing to a one-quarter wavelength phase difference between the interface shape perturbation and the tangential velocity jump. Hence determining the long-time linear growth rate is equivalent to modeling the initial circulation-line density, or vortex-sheet strength, across the interface. As a last remark, it can be shown after some algebra that, within the model presented here, the jump in transverse velocity $u_{1}^{(1)}-u_{2}^{(1)}$ across the interface is not constant with time unless the base flow is symmetric, that is, $-U_{S_{1}}=U_{S_{2}}$. However, the momentum slip $\rho_{1} u_{1}^{(1)}-\rho_{2} u_{2}^{(1)}$ across the interface is conserved with time.

If the circulation related to Richtmyer's asymptotic model is taken to be the initial circulation in the present model, Eq. (25) leads to

$$
\begin{aligned}
& \dot{h}_{\infty}=\mathfrak{F}_{\text {Rich. Circ. }} \dot{h}_{\text {Rich. }}=2 \frac{\left(1-A^{+}\right) U_{S_{2}}-\left(1+A^{+}\right) U_{S_{1}}}{U_{S_{2}}-U_{S_{1}}} \dot{h}_{\text {Rich. }}, \\
& \Delta\left[u^{(1)}\right]=2 \iota k h(0) A^{+} \Delta W .
\end{aligned}
$$

Using the leading order (small angles of incidence $\alpha$ ) circulation $\Gamma_{1}^{\prime}$ deposited by the passage of a shock on a planar interface, given the long expression (2.14) in Samtaney and Zabusky, ${ }^{15}$ Eq. (25) becomes

$$
\begin{aligned}
& \dot{h}_{\infty}=\mathfrak{F}_{\text {Samt. Circ. }} \dot{h}_{\text {Rich. }} \\
& \quad=2 \frac{\left(1-A^{+}\right) U_{S_{2}}-\left(1+A^{+}\right) U_{S_{1}}}{U_{S_{2}}-U_{S_{1}}} \frac{\Gamma_{1}^{\prime}}{2 A^{+} \Delta W} \dot{h}_{\text {Rich. }}, \\
& \Delta\left[u^{(1)}\right]=\iota k h(0) \Gamma_{1}^{\prime} .
\end{aligned}
$$

Subsection 4.4 in Ref. 15 suggests a scaling analysis for $\Gamma_{1}^{\prime}$ in terms of incident Mach number, density ratio (and therefore Atwood ratio), and ratio of specific heats. 


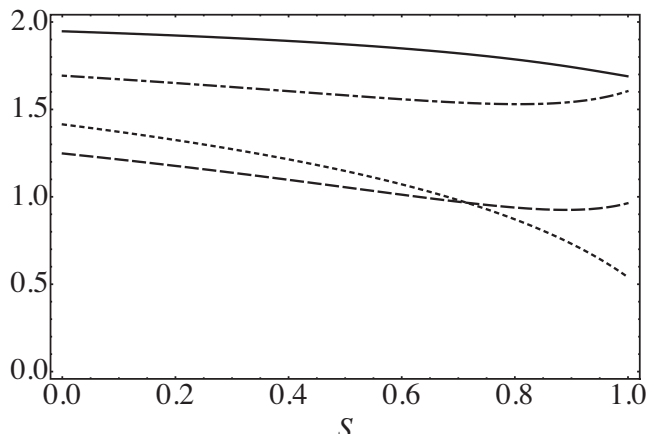

(a) $\mathfrak{F}_{\text {Rich.Circ. }}$ based on Richtmyer's circulation

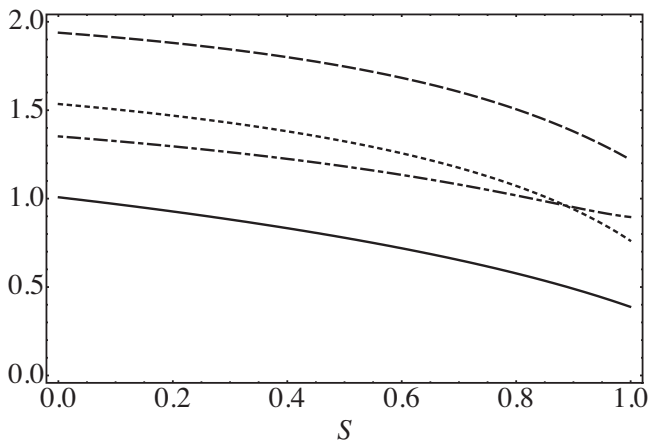

(b) $\mathfrak{F}_{\text {Samt.Circ. }}$ based on Samtaney's

circulation

FIG. 2. Correction factor to Richtmyer's impulsive growth rate for various incident shock strengths $S$ and different combinations of gases: air $\rightarrow \mathrm{CO}_{2}$ (solid line), $\mathrm{Ar} \rightarrow \mathrm{Xe}$ (dot-dashed line), air $\rightarrow \mathrm{SF}_{6}$ (small dashed line), and $\mathrm{He} \rightarrow$ air (long dashed line).

Referring to the form (24), we display in Fig. 2 (and later in Table I) the correction factor $\mathfrak{F}$ determined from Richtmyer's circulation modeling summarized in Eq. (26a), noted $\mathfrak{F}_{\text {Rich. Circ. }}$ and from Samtaney's circulation analysis expressed in Eq. (27a), noted $\mathfrak{F}_{\text {Samt. Circ. In Fig. 2, various com- }}$ mon gases are used, such as air, $\mathrm{Ar}, \mathrm{CO}_{2}, \mathrm{He}, \mathrm{SF}_{6}$, and $\mathrm{Xe}$. A discussion is provided in Sec. IV B. For the comparison against numerical simulations in the following section, the terminal growth rate $\dot{h}_{\infty}$ given by Richtmyer's asymptotic model $\dot{h}_{\text {Rich. }}$ and the correction of Yang et al. $\mathfrak{F}_{\text {Yang }} \dot{h}_{\text {Rich. }}$ will be used. Note that we could have used Wouchuk's results for the asymptotic growth rate since they agree very well with Yang's results. To determine $\dot{h}_{\text {Rich. }}$ we now assume that $h(0)$ in Eq. (23) is the postshock perturbation amplitude $h\left(0^{+}\right)$, which can be evaluated from the numerical simulations presented in the following section.

\section{NUMERICAL SIMULATIONS}

\section{A. Numerical method and diagnostics}

Two-dimensional simulations were conducted within the Adaptive Mesh Refinement Oriented C ++ (AMROC) framework developed by Deiterding ${ }^{16}$ based on the structured adaptive mesh refinement algorithm by Berger and Oliger. ${ }^{17}$ The numerical method, applied to each subgrid of the mesh hierarchy, consists of a hybrid method written for the multicomponent Euler equations of gas dynamics assuming calorically perfect gas: a weighted, essentially nonoscillatory scheme is used to capture discontinuities (such as shock waves, contact wave, or fine/coarse mesh interfaces) but switches to a low-numerical dissipation, explicit, tuned center-difference scheme in the smooth regions. ${ }^{18,19}$

The density interface is nominally defined by the mix-

TABLE I. Various shock-contact interactions for different species and incident shock strengths, obtained with $k h_{0}=0.1 . A^{+}, a_{0} k \tau$, and dimensionless Richtmyer's asymptotic growth rate $k h\left(0^{+}\right) A^{+} \Delta W / a_{0}$ are evaluated by solving the one-dimensional shock-contact interaction problem. $\mathfrak{F}_{\text {Yang }}$ is given by the linearized simulations of Yang et al. (Ref. 8) and represents the exact asymptotic growth rate reached in the linear regime. The models for $\mathfrak{F}_{\text {Rich. Circ. }}$ [given by Eq. (26a)] and $\left.\mathfrak{F}_{\text {Samt. Circ. [given by Eq. }}(27 \mathrm{a})\right]$ are compared to the reference value $\mathfrak{F}_{\text {Yang. }}$.

\begin{tabular}{|c|c|c|c|c|c|c|c|c|c|}
\hline & & $M_{I}$ & $S$ & $A^{+}$ & $a_{0} k \tau$ & $k h\left(0^{+}\right) A^{+}\left(\Delta W / a_{0}\right)$ & $\mathfrak{F}_{\text {Yang }}$ & $\mathfrak{F}_{\text {Rich. Circ. }}$ & $\mathfrak{F}_{\text {Samt. Circ. }}$ \\
\hline $\mathrm{Air} \rightarrow \mathrm{CO}_{2}$ & & 1.2 & 0.339 & 0.222 & 1.280 & 0.0048 & 1.07 & 1.90 & 0.86 \\
\hline$\gamma_{R}=1.40$ & $\gamma_{L}=1.29$ & 1.5 & 0.593 & 0.237 & 1.398 & 0.0092 & 1.14 & 1.85 & 0.72 \\
\hline$A=0.21$ & & 2.0 & 0.778 & 0.253 & 1.493 & 0.0128 & 1.12 & 1.79 & 0.60 \\
\hline $\mathrm{Ar} \rightarrow \mathrm{Xe}$ & & 1.2 & 0.355 & 0.533 & 1.755 & 0.0087 & 1.06 & 1.61 & 1.24 \\
\hline$\gamma_{R}=1.67$ & $\gamma_{L}=1.65$ & 1.5 & 0.610 & 0.527 & 1.812 & 0.0175 & 1.11 & 1.55 & 1.13 \\
\hline$A=0.53$ & & 2.0 & 0.789 & 0.507 & 1.701 & 0.0251 & 1.16 & 1.53 & 1.03 \\
\hline $\mathrm{Air} \rightarrow \mathrm{SF}_{6}$ & & 1.2 & 0.339 & 0.700 & 2.896 & 0.0114 & 1.02 & 1.24 & 1.41 \\
\hline$\gamma_{R}=1.40$ & $\gamma_{L}=1.09$ & 1.5 & 0.593 & 0.732 & 3.726 & 0.0231 & 0.96 & 1.07 & 1.26 \\
\hline \multirow[t]{4}{*}{$A=0.67$} & & 2.0 & 0.778 & 0.766 & 4.792 & 0.0390 & 0.85 & 0.89 & 1.09 \\
\hline & & 3.0 & 0.903 & 0.801 & 5.700 & 0.0669 & 0.71 & 0.72 & 0.92 \\
\hline & & 5.0 & 0.966 & 0.825 & 5.230 & 0.1144 & 0.61 & 0.61 & 0.83 \\
\hline & & 8.0 & 0.987 & 0.835 & 3.914 & 0.2103 & 0.56 & 0.57 & 0.78 \\
\hline $\mathrm{He} \rightarrow$ air & & 1.2 & 0.355 & 0.770 & 3.142 & 0.0100 & 1.01 & 1.11 & 1.81 \\
\hline$\gamma_{R}=1.67$ & $\gamma_{L}=1.40$ & 1.5 & 0.610 & 0.779 & 3.506 & 0.0222 & 1.00 & 1.00 & 1.67 \\
\hline$A=0.76$ & & 2.0 & 0.789 & 0.780 & 3.518 & 0.0401 & 0.56 & 0.57 & 0.78 \\
\hline
\end{tabular}


ture fraction field $\psi(x, z, t)$. This scalar field is asymptotic to $\psi(x, z, t)=0$ on the very right side, $\psi(x, z, t)=1$ on the very left side, and is initially setup using a tanh profile with intrinsic thickness $\delta_{0}^{C}$ (taken as about one fifth of the preshock perturbation amplitude $h_{0}$ ). When the shock impacts the smeared interface, it is compressed down to a thickness $\delta^{C}(t)$ until the end of the shock refraction when both reflected and transmitted shock travels away from the interface. It is important to ensure that during the whole simulation, in particular during the shock interaction, $\delta^{C}$ is fully resolved. The resolution, controlled by the number of refinement levels, is chosen such that at least 10 points are used to resolved the interface thickness.

At $t>0$, we define the centerline of the smeared density interface by

$$
z_{c}(x, t) \equiv\left(\int_{-\infty}^{\infty} z(1-\psi) \psi d z\right) /\left(\int_{-\infty}^{\infty}(1-\psi) \psi d z\right)
$$

Indeed, at a given time $t$, for a fixed $x$, the scalar profile can be well approximated by a tanh centered at $z_{c}(x, t)$ and with thickness $\delta^{C}(t)$,

$$
\psi(x, z, t)=\frac{1}{2}\left(1+\tanh \left\{\frac{2\left[z-z_{c}(x, t)\right]}{\delta^{C}(t)}\right\}\right) .
$$

It is easily verified that $z_{c}(x, t)$ is recovered when Eq. (29) is used in Eq. (28). The spike and the bubble positions and the flow velocity at these locations allow a measurement of perturbation amplitude and growth rate,

$$
\begin{aligned}
& h(t)=\left|\frac{z_{c_{\text {spike }}}-z_{c_{\text {bubble }}}}{2}\right|, \\
& \dot{h}(t)=\left|\frac{\left.w\right|_{z_{c_{\text {spike }}}}-\left.w\right|_{z_{c_{\text {bubble }}}}}{2}\right| .
\end{aligned}
$$

\section{B. Parametric study of the amplitude and growth rate of the interface perturbation}

The parameters involved are the preshock Atwood ratio $A$, the ratio of specific heats $\gamma_{j}$ for each specie, the incident shock Mach number $M_{I}$ (or its strength $S$ ), the perturbation wavenumber $k$, and the preshock perturbation amplitude $h_{0}$. The Atwood ratio is chosen such that the temperature is continuous across the initial interface (which is consistent with experimental conditions), and as a result, $A$ is a function only of the molecular weights of both species. To a given combination of parameters corresponds a numerical simulation from which amplitude and growth rate of the interface are obtained. Simulation data are compared to the theoretical model presented in the previous section in Eq. (17).

We recall that the incident shock strength is given by the ratio of the pressures ahead and behind the incident shock $p_{0}$ and $p_{0^{\prime}}$, or in terms of the incident Mach number,

$$
S=1-\frac{p_{0}}{p_{0^{\prime}}}=1 /\left(1+\frac{\gamma_{R}+1}{2 \gamma_{R}\left(M_{I}^{2}-1\right)}\right) .
$$

In the region to the right of the interface before the shock interaction, the speed of sound

$$
a_{0} \equiv \sqrt{\left(\gamma_{R} p_{0} / \rho_{0}\right)}
$$

is the reference velocity scale in the data representation. $1 /\left(a_{0} k\right)$ is the reference time scale. Table I shows different types of shock-contact interaction (at a fixed $k h_{0}$ ). For each combination of species, at a given Mach number, we compute the postshock Atwood ratio $A^{+}$, the dimensionless time $a_{0} k \tau$, the dimensionless impulsive growth rate $k h\left(0^{+}\right) A^{+} \Delta W / a_{0}$, and the correction factor $\mathfrak{F}$ to Richtmyer's impulsive growth rate using the numerical computations of Yang et al. (see Fig. 16 in Ref. 8), or using a model based on Richtmyer's circulation or Samtaney's initial circulation deposited during the shock interaction. Compared to the reference computations of Yang et al., the model based on Samtaney's circulation appears satisfactory for low Atwood ratios but overestimates the asymptotic growth rate for high Atwood ratios. This is because we used Samtaney's circulation derived for low-density contrasts (see domain of validity in Fig. 15 in Ref. 15). The model based on Richtmyer's circulation overestimates the growth rate for low Atwood ratios but performs very well for higher Atwood ratios and a wide range of Mach numbers as shown in the example of air $\rightarrow \mathrm{SF}_{6}$. Some further effort on modeling are needed in particular because the factor $\left[\left(1-A^{+}\right) U_{S_{2}}-\left(1+A^{+}\right) U_{S_{1}}\right] /\left(U_{S_{2}}\right.$ $\left.-U_{S_{1}}\right)$ does not tend to unity in the limit of weak incident shocks. We note that the postshock amplitudes proved to be almost independent of $k$ over the chosen range of $k$ and Table I presents results obtained with a fixed $k$.

Figure 3 shows the early-time evolution of amplitude and growth rate of the perturbation for a set of parameters indicated in the caption. The model captures well the time scale of the growth. In order to capture the higher-frequency features in detail, which are purely compressible effects due to reverberation of waves between the interface and the bifurcated shocks, a higher-order solution in $\varepsilon_{j}$ would be needed as well as higher-order boundary conditions at shocks.

We now discuss and summarize the influence of the dimensionless parameter $k h_{0}$ without showing relevant figures of the growth amplitude and rate versus time. In the zerointerface thickness limit, there is only one characteristic length scale and varying $h_{0}$ or $k$ is equivalent. However, for a finite $\delta_{0}^{C}$, we must vary independently $h_{0}$ or $k$. Consider, for example, the shock interaction air $\rightarrow \mathrm{SF}_{6}$, as an incident shock of Mach number $M_{I}=1.2$ travels from air to $\mathrm{SF}_{6}$. The initial dimensionless amplitude of the perturbation $k h_{0}$ was varied from 0.03 to 0.3 (varying both $k$ and $h_{0}$ independently). Additional levels of refinement were used in the simulations such that sufficient resolution was provided to capture smaller wavelengths as well as smaller amplitudes of the perturbation. As long as $k h(t)$ remained small compared to $2 \pi$, the growth was observed to be predominantly linear, and good comparisons were obtained between theory and 


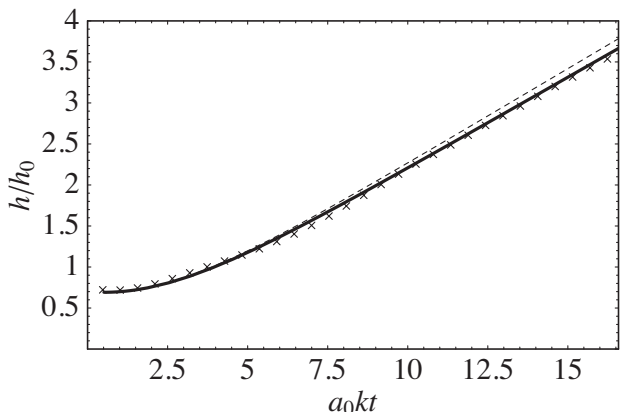

(a) $h(t) / h_{0}$

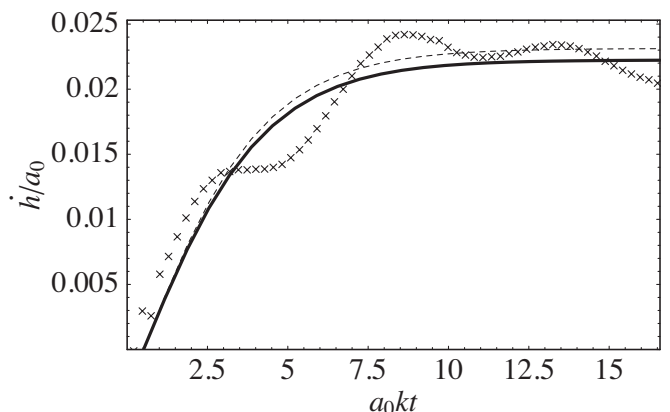

(b) $\dot{h} / a_{0}$

FIG. 3. Early-time closeup: dimensionless amplitude and growth rate of the interface perturbation $h(t) / h_{0}$ and $\dot{h} / a_{0}$ vs $a_{0} k t ;$ case air $\rightarrow \mathrm{SF}_{6}, k h_{0}=0.1, M_{I}$ $=1.5$. Numerical simulations using AMROC are represented by crosses. The thin dashed and thick solid lines correspond to our model given by Eq. (17) with two different choices for $\dot{h}_{\infty}$. The thin dashed line corresponds to Eq. (17) using $\dot{h}_{\infty}=\dot{h}_{\text {Rich. }}$, while the thick solid line uses the correction of Yang et al. to Richtmyer's asymptotic growth rate $\dot{h}_{\infty}=\mathfrak{F}_{\text {Yang }} \dot{h}_{\text {Rich. }}$. The exact expression of $\dot{h}_{\text {Rich. }}$ is given by Eq. (23) and $\mathfrak{F}_{\text {Yang }}$ is provided by the linearized simulations of Yang et al. (Ref. 8). While Eq. (17) gives an explicit expression for the growth rate (up to the knowledge of $\dot{h}_{\infty}$ ), the amplitude of the interface perturbation is obtained by numerically integrating Eq. (17).

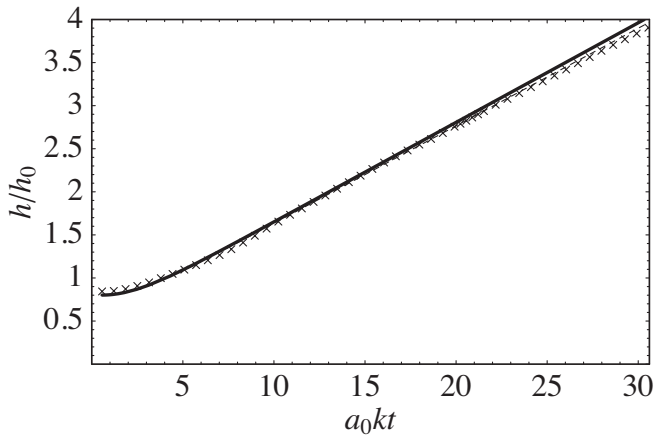

(a) $M_{I}=1.2$

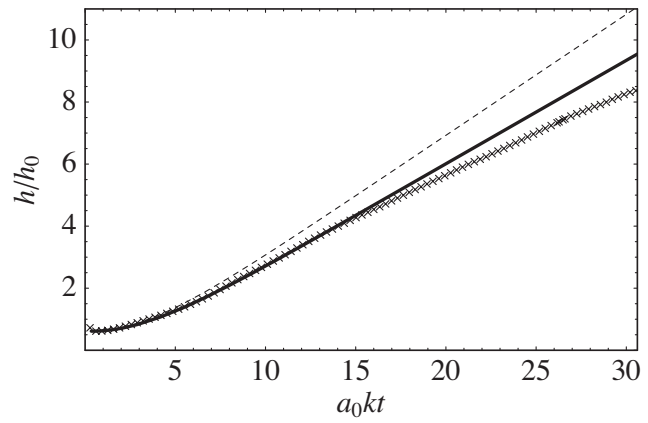

(c) $M_{I}=2.0$

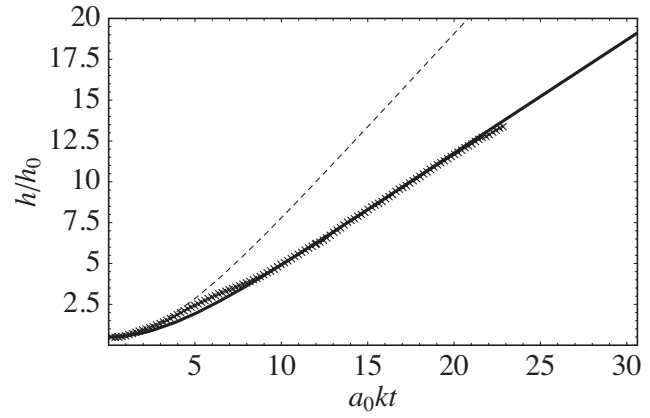

(e) $M_{I}=5.0$

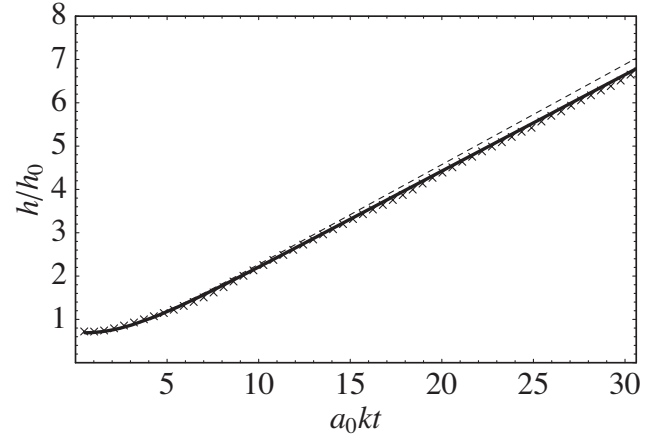

(b) $M_{I}=1.5$

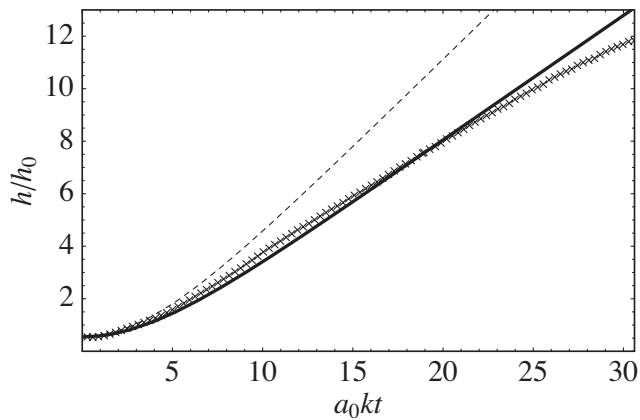

(d) $M_{I}=3.0$

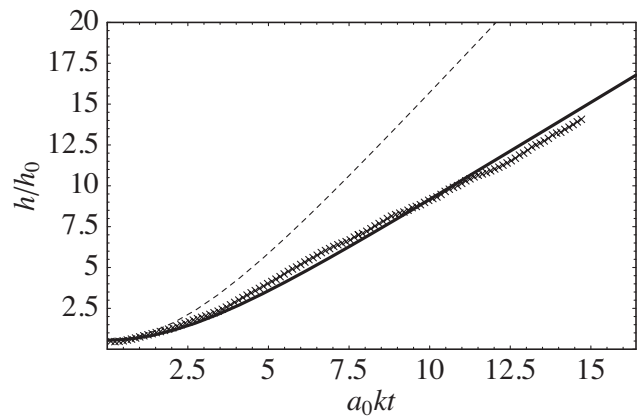

(f) $M_{I}=8.0$

FIG. 4. Influence of $M_{I}$ : dimensionless amplitude of the interface perturbation $h(t) / h_{0}$ vs $a_{0} k t$; case air $\rightarrow \mathrm{SF}_{6}, k h_{0}=0.1$. For key, see Fig. 3 . 


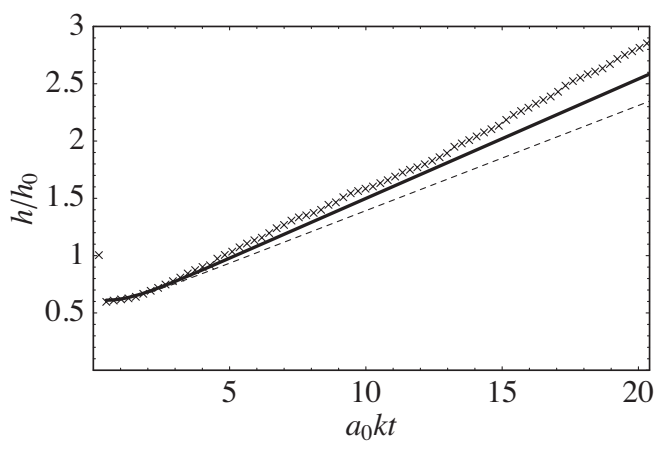

(a) air $\rightarrow \mathrm{CO}_{2}$

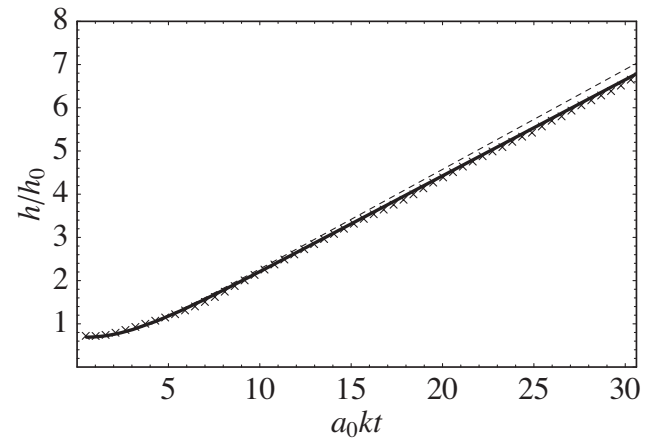

(c)air $\rightarrow \mathrm{SF}_{6}$

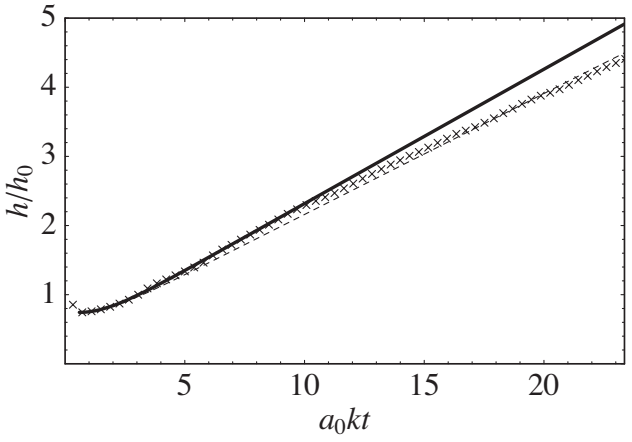

(b) Ar $\rightarrow \mathrm{Xe}$

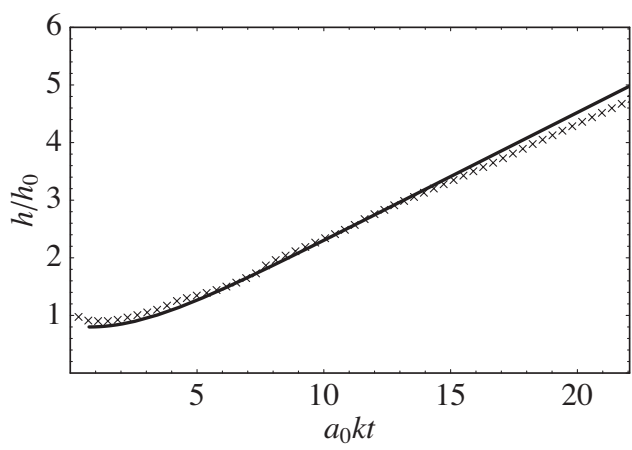

(d)He $\rightarrow$ air

FIG. 5. Influence of the species: dimensionless amplitude of the interface perturbation $h(t) / h_{0}$ vs $a_{0} k t$; case $k h_{0}=0.1, M_{I}=1.5$. For key, see Fig. 3 .

simulation. As $k h_{0}$ increased, the linear growth was found to become faster and to compete earlier with nonlinear growth.

We consider further the case of the air $\rightarrow \mathrm{SF}_{6}$ interaction. The perturbation shape is fixed in amplitude $h_{0}$ and wavenumber $k$, with $k h_{0}=0.1$. The effect of incident shock strength is now studied (see Table I). Figure 4 shows good agreement between the simulated amplitude and that obtained from our model using the correction of Yang et al. for different shock strengths. We insist that the combination air $\rightarrow \mathrm{SF}_{6}$ is a critical test for strong incident shocks where the discrepancy between the model of Richtmyer $\dot{h}_{\text {Rich }}$ significantly overestimates the actual asymptotic growth rate. For very high incident shock strengths, nonlinear slowdown appears earlier and the asymptotic linear growth at late times tends to overestimate the terminal growth obtained in the simulations. It is surprising that the model works rather well even in the strong incident shock case where the interface is accelerated enough that it remains close to the transmitted shock, yet the incompressible model does not include the direct coupling between interface and shock perturbations.

The differences between various gas combinations are reported in Figs. 5 and 6. An incident shock of moderate Mach number $M_{I}=1.5$ impacts a density interface of characteristics $h_{0}$ and $k$ fixed such that $k h_{0}=0.1$. The main characteristics of these interactions are reported in Table I. Perturbation amplitude versus time is represented in Fig. 5. As shown in Fig. 6, the model summarizes well the simulated growth rate. The growth rate computed from the simulations exhibits a different structure depending on the combination of species chosen. Indeed, the wave reverberation frequency on either side of the interface depends on the sound celerity in the related medium, which depends on the local postshock density field and specific heat ratio. The influence of the Atwood ratio and specific heat ratios on the high-frequency oscillations and the growth of the instability at later time are left for future work.

\section{ON THE TIME SCALE $\tau$}

In this section we investigate the dependence of $\tau$, given by Eq. (22), on the flow parameters sufficient to describe the RMI. The time scale $\tau$ represents the characteristic time for the growth rate to attain the constant asymptotic growth rate predicted by the present linearized model. This asymptotic growth generally persists for a further time period, which may be long compared with $\tau$, and is then followed by the onset of the nonlinear regime when the amplitude of the perturbation has increased and the growth rate begins to slow. Nonlinear effects are expected for high $k, A$, or $M_{I}$ (thus $\Delta W$ ), as appearing, for example, in Fig. 4(c).

In the present theory, the dimensionless time $a_{0} k \tau$ depends on the postshock Atwood number $\mathrm{A}^{+}$and the reflected shock speeds $U_{S_{1}}$ and $U_{S_{2}}$. These quantities, in turn, are functions of the preshock Atwood number $A(-1<A<1)$, the incident shock Mach number $M_{I}$, or equivalently, its shock strength $S(0<S<1)$, and the specific heat ratios $\gamma_{R}$ (for the fluid on the right of the interface) and $\gamma_{L}$ (on the left). They can be computed by solving the one-dimensional Riemann problem at $t=0$ for the range of parameters that admit a reflected-shock solution. Presently we consider $a_{0} k \tau$ as a 


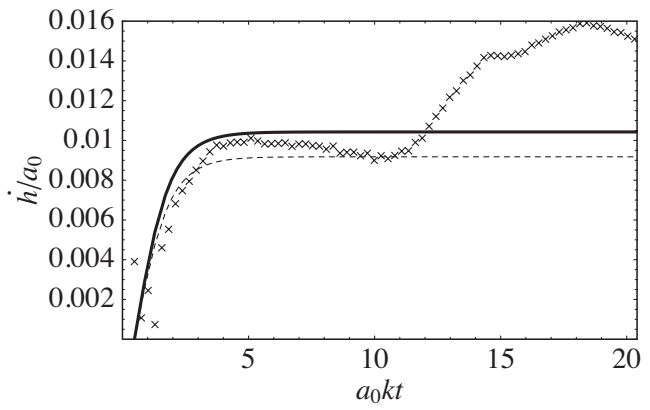

(a) air $\rightarrow \mathrm{CO}_{2}$

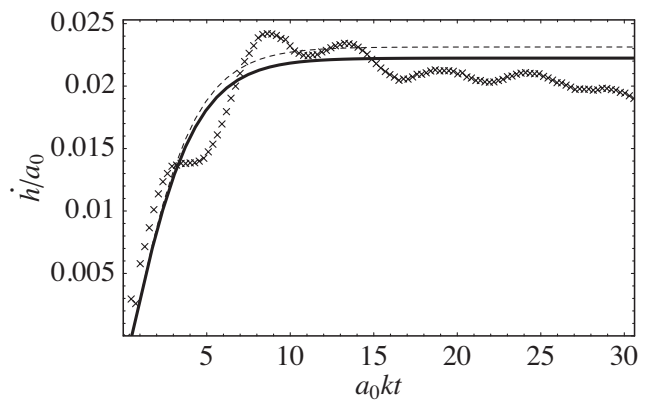

(c) air $\rightarrow \mathrm{SF}_{6}$

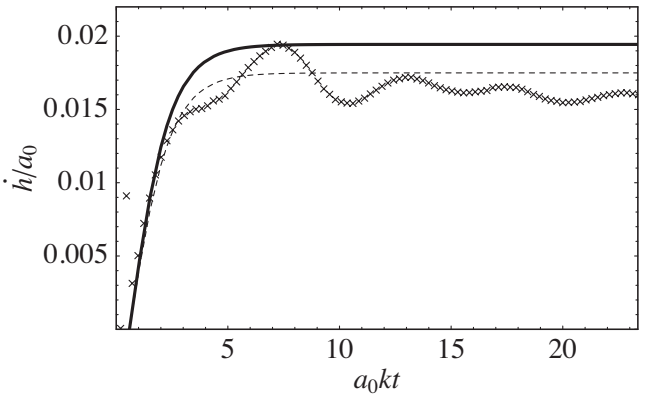

(b) $\mathrm{Ar} \rightarrow \mathrm{Xe}$

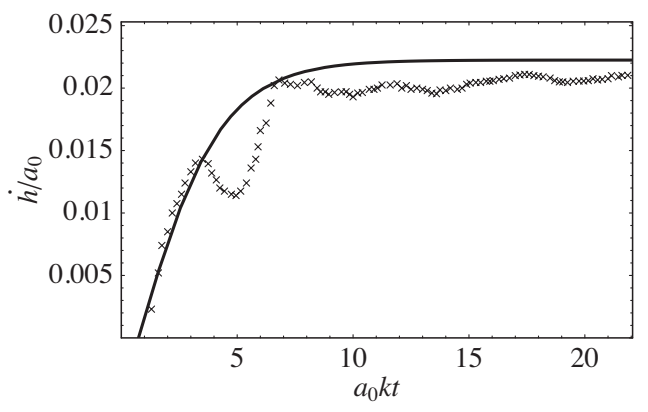

(d) $\mathrm{He} \rightarrow$ air

FIG. 6. Influence of the species: dimensionless growth rate of the interface perturbation $\dot{h} / a_{0}$ vs $a_{0} k t$; case $k h_{0}=0.1, M_{I}=1.5$. For key, see Fig. 3 .

function of $(A, S)$ for a given $\gamma_{L}, \gamma_{R}$. If it is assumed that, for $t<0$, the interface is both temperature and pressure matched, then $A$ is determined uniquely by the ratio of molecular weights of the species separated by the interface (see, for example, Table I).

\section{A. Domain of validity in the case of a reflected shock}

Because we presently restrict attention to the reflectedshock case, it is useful to consider the domain of validity for this case in terms of the parameters that determine $a_{0} k \tau$. A $(p-w)$-diagram analysis enables the determination of the reflected wave type produced when a plane shock impacts a plane interface, depending on the parameters $A, S, \gamma_{R}$, and $\gamma_{L}$. Generally, a reflected shock is produced if the acoustic impedance on the right of the interface $\rho_{R} a_{R}$ is less than the left one $\rho_{L} a_{L}$, that is,

$$
A>\frac{\gamma_{R}-\gamma_{L}}{\gamma_{R}+\gamma_{L}}
$$

If $\gamma_{R}=\gamma_{L}$, this is simply $A>0$, which refers to the "light-toheavy" denomination. Anomalous reflection can actually occur in the case $\gamma_{R} \neq \gamma_{L}$ for particular incident shock strengths. Omitting the detailed analysis of the shock-contact interaction problem, we summarize the conditions for reflected shock in Table II. The critical incident shock strength $S^{*}$ beyond which the reflected wave type changes depends on $\Lambda$ as

$$
S^{\star}(A)=2 \frac{\gamma_{R}-\gamma_{L}-\left(\gamma_{R}+\gamma_{L}\right) A}{\gamma_{R}-\gamma_{L}-\left(\gamma_{R}+\gamma_{L}-2\right) A} .
$$

For real gases, $\gamma_{R}$ and $\gamma_{L}$ are sufficiently close that the change in the structure of the reflected wave occurs at relatively small Atwood ratios.

\section{B. Parametric study of $\tau$}

For the case of a reflected shock, the dimensionless startup time $a_{0} k \tau$ was computed numerically as a function of $(A, S)$ for several sets of $\gamma_{L}, \gamma_{R}$. Results are shown in Fig. 7. $\tau$ increases with $A$ (at fixed $S$ ) and reaches its highest values for high incident shock strengths $S$. These conditions correspond to the situation where the accelerated interface follows closely the transmitted shock. Pressure waves actively move back and forth between the interface and the shock, and $\tau$, which was determined from an incompressible analysis, can

TABLE II. Conditions, in the $(A, S)$ space, for the reflected wave to be a shock wave.

\begin{tabular}{llll}
\hline \hline$\gamma_{R}=\gamma_{L}:$ & & $\forall A \in] 0,1[$, & \\
$\gamma_{R}<\gamma_{L}:$ & & $\forall A \in]\left(\gamma_{R}-\gamma_{L}\right) /\left(\gamma_{R}+\gamma_{L}+2\right), 1[$, & \\
& or & $\forall A \in]\left(\gamma_{R}-\gamma_{L}\right) /\left(\gamma_{R}+\gamma_{L}\right),\left(\gamma_{R}-\gamma_{L}\right) /\left(\gamma_{R}+\gamma_{L}+2\right)[$, & $S \in] 0, S^{\star}(A)[$ \\
$\gamma_{R}>\gamma_{L}:$ & & $\forall A \in]\left(\gamma_{R}-\gamma_{L}\right) /\left(\gamma_{R}+\gamma_{L}\right), 1[$, & $S \in] S^{\star}(A), 1[$ \\
& or & $\forall A \in]\left(\gamma_{R}-\gamma_{L}\right) /\left(\gamma_{R}+\gamma_{L}+2\right),\left(\gamma_{R}-\gamma_{L}\right) /\left(\gamma_{R}+\gamma_{L}\right)[$, & \\
\hline \hline
\end{tabular}




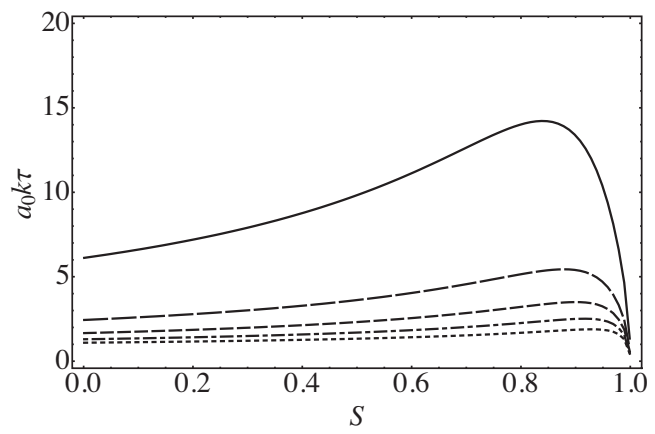

(a) $\gamma_{R}=\gamma_{L}=1.1$

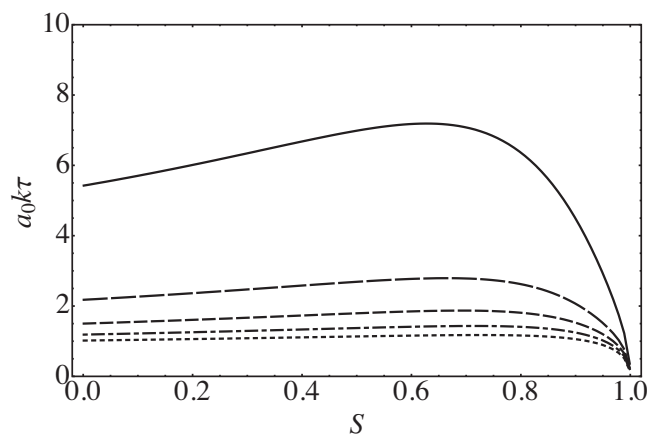

(c) $\gamma_{R}=1.1<\gamma_{L}=1.4$

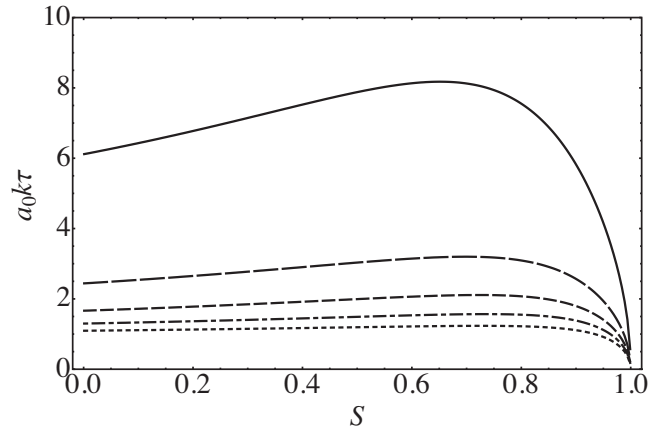

(b) $\gamma_{R}=\gamma_{L}=1.4$

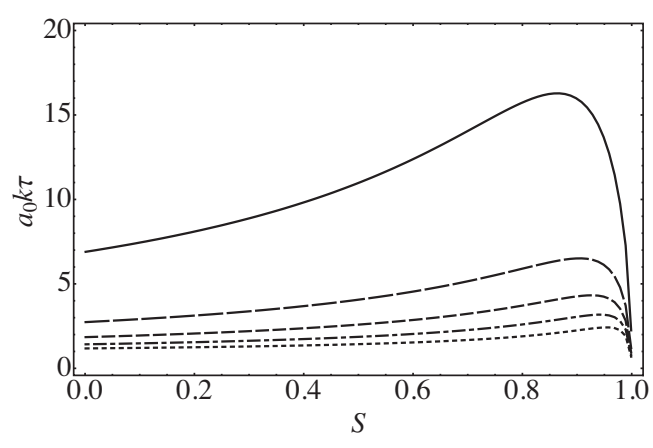

(d) $\gamma_{R}=1.4>\gamma_{L}=1.1$

FIG. 7. $a_{0} k \tau$ vs $S$ for $A=0.15$ (small dashed line), 0.35 (dot-dashed line), 0.55 (long dashed line), 0.75 (very long dashed line), and 0.95 (solid line).

be understood as the result of an averaging of these reverberating waves adding or removing baroclinic vorticity at their passage through the interface. This idea is validated by the various comparisons against the numerical simulations shown in the precedent section.

We investigate the weak incident shock limit $S \rightarrow 0^{+}$. On performing a straightforward analysis of the one-dimensional shock-contact interaction in this limit, it is found that both reflected and transmitted shocks are weak. The postshock Atwood ratio $A^{+}$tends to the preshock Atwood ratio $A$, and the shock speeds in the frame of the moving interface tend to the preshock sound speeds on each respective side of the interface. These observations lead to

$$
a_{0} k \tau=\frac{(1-A)^{3 / 2}+\sqrt{\gamma_{R} / \gamma_{L}}(1+A)^{3 / 2}}{2 \sqrt{1-A}}+\mathcal{O}(S) \quad \text { as } S \rightarrow 0^{+} .
$$

The dominant term of $\tau$ is independent of $S$. In particular, in this limit, as $A \rightarrow 0^{+}$and if $\gamma_{R}=\gamma_{L}, a_{0} k \tau \rightarrow 1$. As $A \rightarrow 1^{-}, \tau$ naturally becomes infinite.

The strong shock limit $S \rightarrow 1^{-}$is now analyzed. As the incident shock becomes stronger, the transmitted shock strength increases accordingly, while the Mach number of the reflected shock wave converges to a finite number $M_{R_{*}}$. The postshock Atwood ratio tends to a finite value $A_{*}^{+}$. Finally $\tau$ is given by

$$
\begin{aligned}
a_{0} k \tau= & \frac{1}{2} \sqrt{\frac{\gamma_{R}+1}{\gamma_{R}-1}}\left(\frac{1-A_{*}^{+}}{M_{R_{*}}-\left[2 /\left(\gamma_{R}+1\right)\right]\left[\left(M_{R_{*}}^{2}-1\right) / M_{R_{*}}\right]}\right. \\
& \left.\left.+\frac{1+A_{*}^{+}}{\left(\gamma_{L}-1\right)\left\{\left[2 \gamma_{R}\left(\gamma_{R}-1\right)\right]^{-1 / 2}-\left[1 /\left(\gamma_{R}+1\right)\right]\left[\left(M_{R_{*}}^{2}-1\right) / M_{R_{*}}\right]\right.}\right\}\right) \\
& \times \sqrt{1-S}+\mathcal{O}\left[(1-S)^{n}\right] \text { as } S \rightarrow 1^{-},
\end{aligned}
$$

with $n>1 / 2$ a priori and where $A_{*}^{+}$is a function of $M_{R_{*}}$ of the form 


$$
A_{*}^{+}=\frac{1-\left(\frac{1-A}{1+A}\right)\left(\frac{\gamma_{R}+1}{\gamma_{R}-1}\right)\left(\frac{\gamma_{L}-1}{\gamma_{L}+1}\right) M_{R_{*}}^{2}\left(1+\frac{\gamma_{R}-1}{\gamma_{R}+1}\left(M_{R_{*}}^{2}-1\right)\right)^{-1}}{1+\left(\frac{1-A}{1+A}\right)\left(\frac{\gamma_{R}+1}{\gamma_{R}-1}\right)\left(\frac{\gamma_{L}-1}{\gamma_{L}+1}\right) M_{R_{*}}^{2}\left(1+\frac{\gamma_{R}-1}{\gamma_{R}+1}\left(M_{R_{*}}^{2}-1\right)\right)^{-1}},
$$

and $M_{R_{*}}$ is itself a function of $\left(\gamma_{R}, \gamma_{L}, A\right)$ obtained from solving the following polynomial equation:

$$
\left(1+\frac{2 \gamma_{R}}{\gamma_{R}+1}\left(M_{R_{*}}^{2}-1\right)\right)-\left(\frac{1+A}{1-A}\right)\left(\frac{\gamma_{L}+1}{\gamma_{R}+1}\right)\left(1-\frac{\sqrt{2 \gamma_{R}\left(\gamma_{R}-1\right)}}{\gamma_{R}+1} \frac{M_{R_{*}}^{2}-1}{M_{R_{*}}}\right)^{2}=0 .
$$

For any $A<1, \tau \rightarrow 0$ as $S \rightarrow 1^{-}$. The sublimit $A \rightarrow 1^{-}$is more difficult to investigate here because of the competition between the strong shock limit that tends to decrease $\tau$ and the high Atwood number limit that would increase $\tau$.

Finally, we consider the limit case $A \rightarrow 1^{-}$, or $\rho_{L} \gg \rho_{R}$ (the extreme example would be gas-to-liquid RMI). The shock interaction compressing the heavy fluid, the postshock Atwood ratio also tends to $1^{-}$. Moreover, the shock speed naturally becomes smaller and smaller on the heavy side, such that in Eq. (22), the second term is dominant, and $\tau \sim 1 /\left[k\left(-U_{S_{1}}\right)\right]$. Since $-U_{S_{1}}$ is the following function of the transmitted shock Mach number $M_{T}$ :

$$
-U_{S_{1}}=a_{0} \sqrt{\frac{\gamma_{L}}{\gamma_{R}}} \sqrt{\frac{1-A}{1+A}}\left(M_{T}-\frac{2}{\gamma_{L}+1} \frac{M_{T}^{2}-1}{M_{T}}\right),
$$

where $M_{T}$ converges to a finite value $M_{T_{* *}}$ as $A \rightarrow 1^{-}\left(M_{T_{* *}}\right.$ is obtained by solving numerically the one-dimensional Riemann problem for a given shock strength $S$ ), we then have

$$
\begin{aligned}
a_{0} k \tau= & \frac{\sqrt{2 \gamma_{R} / \gamma_{L}}}{M_{T_{* *}}-\left[2 /\left(\gamma_{L}+1\right)\right]\left[\left(M_{T_{* *}}^{2}-1\right) / M_{T_{* *}}\right]} \frac{1}{\sqrt{1-A}} \\
& +\mathcal{O}\left(\frac{1}{(1-A)^{m}}\right) \quad \text { as } A \rightarrow 1^{-}
\end{aligned}
$$

with $m<1 / 2$. Therefore, in the limit $A \rightarrow 1^{-}, \tau \rightarrow \infty$. In other words, according to our model, the amplitude of small perturbations across a light-to-heavy density interface with very high Atwood number grows quadratically in time, and not linearly as would have predicted Richtmyer's impulsive model. The limit $A \rightarrow 1^{-}$is valid for $S<1$. In particular, as $S \rightarrow 0^{+}$,

$$
a_{0} k \tau \sim \sqrt{2 \frac{\gamma_{R}}{\gamma_{L}}} \frac{1}{\sqrt{1-A}}
$$

which we can recover by taking the limit $A \rightarrow 1^{-}$in Eq. (35). As mentioned earlier, the double limit $(A, S) \rightarrow\left(1^{-}, 1^{-}\right)$ shows a competition between the strong shock and high Atwood number influences. Indeed, as $S \rightarrow 1^{-}$in Eq. (40),

$$
a_{0} k \tau \sim \sqrt{2 \frac{\gamma_{R}}{\gamma_{L}}} \frac{\gamma_{L}+1}{\gamma_{L}-1} \frac{1}{\sqrt{1-A}} \frac{1}{M_{T_{* *}}},
$$

where $M_{T_{* *}} \rightarrow \infty$ as $S \rightarrow 1^{-}$. This last expression is validated by computing the general expression of $\tau$ from the onedimensional shock-interface Riemann problem at very high Atwood ratios and shock strengths.

The analysis of $\tau$ for small $A$ is more difficult to investigate since the nature of the reflected wave can change in this region, as mentioned in Sec. V A.

\section{CONCLUSION}

A simple model capturing the time evolution of the linearized initial growth of the RMI has been developed in the case of a reflected shock, which corresponds in general to light-to-heavy shock interactions. Our main result is the Richtmyer-Meshkov startup time scale given by Eq. (22). We have shown how this time scale can be related to conditions prior to shock-interface impact in the space of the shock strength $S$ and the preshock Atwood ratio $A$. Comparisons have been made of the present theory against numerical simulations for a wide range of $A$ and $S$. A degree of freedom appearing in the analysis allows for additional modeling of the baroclinic vorticity deposition. Several scenarios for corrections to the asymptotic growth rate of Richtmyer have been investigated with emphasis on strong incident shock conditions. Extensions to the present analysis could lead to improved understanding of the linear RMI in more complex applications such as imploding waves propagating into a stratified medium.

\section{ACKNOWLEDGMENTS}

This work was supported by the Advanced Simulation and Computing (ASC) Program under Subcontract No. B341492 of DOE Contract No. W-7405-ENG-48. M.L. and D.I.P. would like to thank Dr. David J. Hill for numerous discussions and comments on the manuscript.

${ }^{1}$ R. D. Richtmyer, "Taylor instability in shock acceleration of compressible fluids," Commun. Pure Appl. Math. 13, 297 (1960).

${ }^{2}$ M. Vandenboomgaerde, C. Mügler, and S. Gauthier, "Impulsive model for the Richtmyer-Meshkov instability," Phys. Rev. E 58, 1874 (1998).

${ }^{3}$ R. L. Holmes, G. Dimonte, B. Fryxell, M. L. Gittings, J. W. Grove, M. Schneider, D. H. Sharp, A. L. Velikovich, R. P. Weaver, and Q. Zhang, 
"Richtmyer-Meshkov instability growth: Experiment, simulation and theory," J. Fluid Mech. 389, 55 (1999).

${ }^{4}$ O. A. Hurricane, E. Burke, S. Maples, and M. Viswanathan, "Saturation of Richtmyer's impulsive model," Phys. Fluids 12, 2148 (2000).

${ }^{5}$ S. G. Glendinning, J. Bolstad, D. G. Braun, M. J. Edwards, W. W. Hsing, B. F. Lasinski, H. Louis, A. Miles, J. Moreno, T. A. Peyser, B. A. Remington, H. F. Robey, E. J. Turano, C. P. Verdon, and Y. Zhou, "Effect of shock proximity on Richtmyer-Meshkov growth," Phys. Plasmas 10, 1931 (2003).

${ }^{6}$ G. Fraley, "Rayleigh Taylor stability for a normal shock wave-density discontinuity interaction," Phys. Fluids 29, 376 (1986).

${ }^{7}$ J. G. Wouchuk, "Growth rate of the linear Richtmyer-Meshkov instability when a shock is reflected," Phys. Rev. E 63, 056303 (2001).

${ }^{8}$ Y. Yang, Q. Zhang, and D. H. Sharp, "Small amplitude theory of Richtmyer-Meshkov instability," Phys. Fluids 6, 1856 (1994).

${ }^{9}$ J. Griffond, "Linear interaction analysis for Richtmyer-Meshkov instability at low Atwood numbers," Phys. Fluids 18, 054106 (2006).

${ }^{10} \mathrm{D}$. Layzer, "On the gravitational instability of two superposed fluids in a gravitational field," Astrophys. J. 122, 1 (1955).

${ }^{11}$ J. Hecht, U. Alon, and D. Shvarts, "Potential flow models of RayleighTaylor and Richtmyer-Meshkov bubble fronts," Phys. Fluids 6, 4019 (1994).

${ }^{12}$ Y. Srebro, Y. Elbaz, O. Sadot, L. Arazi, and D. Shvarts, "A general buoyancy-drag model for the evolution of the Rayleigh-Taylor and Richtmyer-Meshkov instabilities," Laser Part. Beams 21, 347 (2003).

${ }^{13}$ L. D. Landau and E. M. Lifshitz, Fluid Mechanics, 2nd ed. (Pergamon, New York, 1987).

${ }^{14}$ S. P. Dýakov, "The interaction of shock waves with small perturbations," Sov. Phys. JETP 33, 948 (1957).

${ }^{15}$ R. Samtaney and N. J. Zabusky, "Circulation deposition on shockaccelerated planar and curved density-stratified interfaces: Models and scaling laws," J. Fluid Mech. 269, 45 (1994).

${ }^{16}$ R. Deiterding, "Construction and application of an AMR algorithm for distributed memory computers," Adaptive Mesh Refinement Theory and Applications, Lecture Notes in Computational Science and Engineering Vol. 41, edited by T. Plewa, T. Linde, and V. G. Weirs (Springer, Berlin, 2005), pp. 361-372.

${ }^{17}$ M. J. Berger and J. Oliger, "Adaptive mesh refinement for hyperbolic partial-differential equations," J. Comput. Phys. 53, 484 (1984).

${ }^{18}$ D. J. Hill and D. I. Pullin, "Hybrid tuned center-difference-WENO method for large eddy simulations in the presence of strong shocks," J. Comput. Phys. 194, 435 (2004).

${ }^{19}$ C. Pantano, R. Deiterding, D. J. Hill, and D. I. Pullin, "A low numerical dissipation patch-based adaptive mesh refinement method for large-eddy simulation of compressible flows," J. Comput. Phys. 221, 63 (2007). 